\title{
Needle aspiration techniques in the diagnosis of pneumonia
}

\author{
Federico Manresa, Jordi Dorca
}

There is a need for improved diagnostic methods in pneumonia in view of the low bacteriological yield from conventional diagnostic methods (Gram stain and culture of bronchial secretions, pleural fluid and blood culture), the difficulty of identifying the cause of common pulmonary infections on clinical grounds, the increasing number of bacteria that are resistant to commonly used broad spectrum antibiotics (which complicates an empirical approach to treatment), and the clinical importance of correctly identifying the causative organisms of pulmonary infection and favourable cost-benefit ratio. ${ }^{12}$

The aim of needle aspiration is to obtain a respiratory sample that is not contaminated by oropharyngeal flora, the isolation of the organisms causing pulmonary infections thus being permitted.

Of the different needle aspiration techniques that have been introduced to obtain an aetiological diagnosis of pulmonary infections, only two are considered to be of clinical value, transtracheal needle aspiration and transthoracic needle aspiration.

Before any needle aspiration procedure patients should have their prothrombin time and platelet count checked. Contraindications to needle biopsy are detailed below.

\section{The techniques}

TRANSTRACHEAL NEEDLE ASPIRATION

Transtracheal needle aspiration was introduced by Pecora in 1959 and modified by Kalinske et $a l^{3}$ It is carried out as follows (fig 1):

Servei de

Pneumologia,

Hospital de Bellvitge,

University of

Barcelona, 08907

L'Hospitalet del

Llobregat, Barcelona,

Spain

F Manresa

J Dorca

Reprint requests to:

Dr Manresa

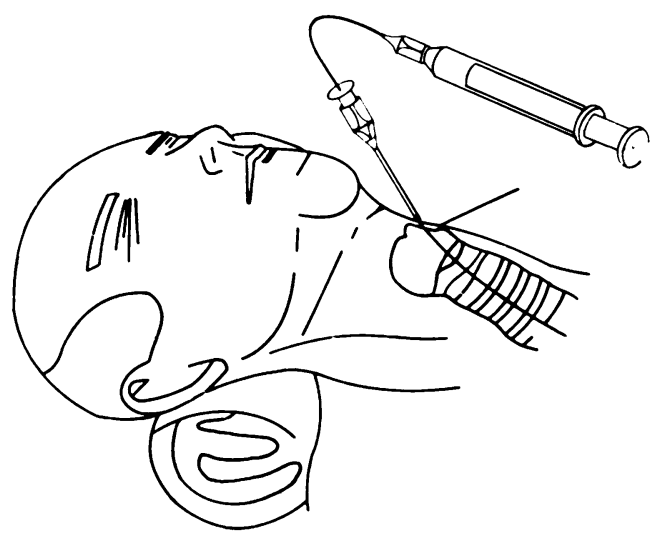

The technique of transtracheal needle aspiration.
The patient is placed in the supine position with a cushion beneath his shoulders, so that the neck is hyperextended. After the cricothyroid membrane has been located local anaesthetic (5 ml mevipacaine HCL) is applied from the skin to the outside of the trachea. The trachea is punctured (fig 1) by a needle guiding a venous catheter (Venocath 14G, Venisystems Abbott, Sligo, Ireland), with a caudal angle of $45^{\circ}$. The catheter, inside the needle, is then pushed into the trachea to a distance of at least $10 \mathrm{~cm}$. Once the catheter is in the trachea the needle is withdrawn. During this last procedure the trachea should be compressed gently for four minutes to avoid subcutaneous emphysema.

Respiratory secretions are then obtained by aspirating the catheter through a Lukens container. To improve the specificity of the technique quantitative or semiquantitative cultures are needed. The instillation of saline into the trachea is not recommended because it will dilute the sample and may thus interfere with the quantitative assessment.

After withdrawal of the catheter the cervical region must be compressed for several minutes.

\section{TRANSTHORACIC NEEDLE ASPIRATION}

Transthoracic needle aspiration (fig 2) is the oldest technique used to determine the aetiology of pulmonary infections. Since 1980 ultrathin needles have been used for diagnostic purposes for pneumonia and neoplasia to minimise the occurrence of pneumothorax. ${ }^{4}$

The pulmonary consolidation must be located correctly. The patient is then placed in position to facilitate the introduction of the needle directly into the lung lesion. The procedure is similar to that of a pleural tap and can be carried out at the bedside.

Local anaesthesia is injected into the chest wall, as for a pleural tap. A large, short guide needle (fig 2 ) is passed into the parietal pleura. The ultrathin needle with its inner stylet $(15.0 \mathrm{~cm}$ long, Cook DZLB 25G, William Cook Europe, Sandet 6 Bjaeverskov, Denmark) is then introduced through the large guide needle into the consolidated lung. The patient is instructed to breath shallowly, gently, and slowly while the long thin needle is passed into the lung parenchyma. After removal of the stylet a syringe containing $5 \mathrm{ml}$ saline is connected to the needle; $4 \mathrm{ml}$ of saline is injected into the lesion and immediately withdrawn, 


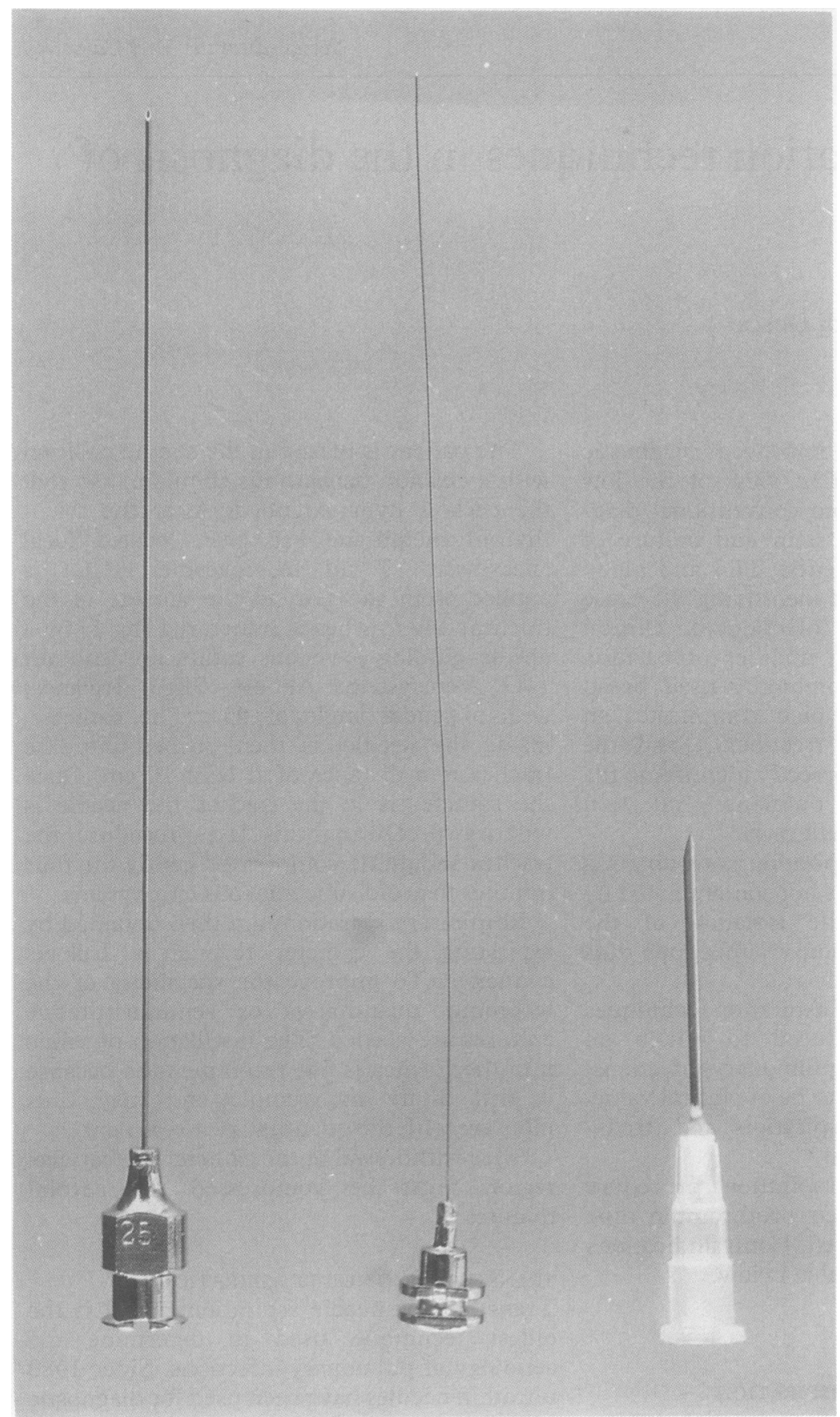

Figure 2 From left to right: needle, stylet, and large guide needle.
Diagnostic value and safety

The diagnostic value of the transtracheal needle aspiration is remarkably high; its overall sensitivity in bacterial pneumonia and anaerobic pulmonary infections has reached $80 \%$ in certain series. ${ }^{56}$ Its specificity is reported to be low in some circumstances, however, about $22 \%$ of patients with lung cancer and $40 \%$ of patients with chronic bronchitis (frequent hosts of organisms causing bacterial pneumonia) having false positive results due to contamination of the bronchial tree. ${ }^{78}$ These figures are even higher in patients with gastric aspiration or in coma.

Complications from this technique are rare, the most important being subcutaneous haemorrhage and emphysema, which occur in less than $10 \%$ of cases. Vagal reactions, cervical cellulitis, angina pectoris, and spasm of the glottis have been reported. ${ }^{10}$

Transtracheal aspiration is not very acceptable to patients and other procedures (protected bronchoscopic specimen brushing and transthoracic needle aspiration) are replacing it. The number of series in which this technique has been used has declined substantially in the last five years. ${ }^{11}$

The sensitivity of transthoracic needle aspiration has varied from $60 \%$ to $90 \%$, depending on the type of infection. Several authors have found the specificity to be almost $100 \%$, and for practical purposes the organism isolated after Gram staining or culture of a transthoracic needle aspirate (excluding cutaneous contaminants such as coagulase negative staphylococci etc) is considered to be responsible for the pneumonia. ${ }^{412-14}$

With the use of ultrathin needles the complication rate of this technique is extremely low. In our experience of more than 300 procedures only $1.2 \%$ of patients have had complications that required treatment (pneumothorax or haemoptysis). ${ }^{12}$ Patients' acceptance of the procedure is good and similar to that of a thoracocentesis.

The training required to perform any of these aspiration techniques correctly is very simple. The low incidence of complications may be attributed to adequate training, the simplicity of the procedure, and the type of needle used.

\section{Indications and contraindications}

The two needle techniques have identical indications but, as mentioned earlier, transthoracic needle aspiration (and use of the bronchoscope protected specimen brush) will probably replace transtracheal aspiration for the diagnosis of pulmonary infections in patients breathing spontaneously.

In our experience transthoracic aspiration complements conventional methods of investigation (Gram staining of bronchial secretions, blood culture, etc), and we consider its use in the following clinical settings:

1 hospital acquired pulmonary infections in non-ventilated patients;

2 community acquired pneumonia that may be caused by an uncommon pathogen (in old 
age, chronic debilitating diseases, immunosuppression, etc);

3 pneumonia that fails to respond to appropriate empirical treatment;

4 lung abscesses not responding to apparently appropriate empirical treatment.

The principal contraindications for the needle techniques are coagulation disturbances: thrombocytopenia (the lowest acceptable platelet count is $100000 / \mathrm{ml}$ and a prothrombin time of $60 \%$. Other contraindications are untreatable cough, very poor lung function $\left(\mathrm{FEV}_{1}\right.$ below $\left.1.0 \mathrm{l}\right)$, and the presence of bullae.

We do not recommend the use of transthoracic needle aspiration in patients undergoing assisted ventilation.

1 Research Committee of the British Thoracic Society and the Public Health Laboratory Service. Community acquired pneumonia in adults in British hospitals in 1982-83: a survey of aetiology, mortality, prognostic factors and outcome. $O J$ Med 1987;239:195-200.

2 Dorca J, Ruf G, Verdaguer R, et al. Therapeutical relevance of the transthoracic aspiration with ultrathin needle in bacterial lung infections [abstract]. Am Rev Respir Dis 1990;141:A861.
3 Kalinske R, Parker R, Bandt D, Hoeprich P. Diagnostic usefulness and safety of transtracheal aspiration. N Engl J Med 1967;276:604-8.

4 Zavala DC, Schoell JE. Ultrathin needle aspiration of the lung in infectious and malignant diseases. Am Rev Respir Dis 1981;123:125-31.

5 Irvin R, Pratter M. Transtracheal aspiration procedure: a protocol. Chest 1981;79:245-7.

6 Ries $\mathrm{K}$, Levison M, Kaye D. Transtracheal aspiration in pulmonary infection. Arch Intern Med 1974;133:453-8.

7 Bjerkestrand G, Digranes A, Achreiner A. Bacteriological findings in transtracheal aspirates from patients with
chronic bronchitis and bronchiectasis. Scand J Respir Dis 1975;56:201-7.

8 Fossieck B, Parker RH, Cohen MH, Kane RC. Fiberoptic bronchoscopy and culture from the lower respiratory tract. Chest 1977;72:5-9.

9 Bartlett JG, Rosenblatt J, Finegold S. Percutaneous transtracheal aspiration in the diagnosis of anaerobic pulmonary infection. Ann Intern Med 1973;79:535-58.

10 Irwin R, Pratter M. Transtracheal aspiration guidelines for your safety. Chest 1979;76:518-20.

11 Manresa F. Rapid clinical diagnostic methods in respiratory infections. Current Opinion Infect Dis 1989;2:536-40.

12 Dorca J, Boada J, Verdaguer R, et al. The transthoracic aspiration with ultrathin needle in the diagnosis of bacterial pulmonary infection [abstract]. Eur Respir $J$ 1988;1 (suppl 2):264S.

13 Palmer DL, Davidson M, Lusk R. Needle aspiration of the lung in complex pneumonia. Chest 1980;78:16-21.

14 Castellino RA, Blank N. Etiologic diagnosis of focal pulmonary infection in immunocompromised patients by fluoroscopically guided percutaneous needle aspiration. Radiology 1979;132:563-7. 